\title{
Synthesis of 2D Hexagonal Mesoporous Silica Using Amino Acid-based Surfactant Templating
}

\author{
Hailan $\mathrm{XU}^{1}$, Huan $\mathrm{YANG}^{3}$, Guangtong $\mathrm{XU}^{2}$ and Yuxiang $\mathrm{YANG}^{3, \mathrm{a}^{*}}$ \\ ${ }^{1}$ School of Chemical and Environmental Engineering, Shanghai Institute of Technology, Shanghai \\ 200235, China \\ ${ }^{2}$ State Key Laboratory of Catalytic Materials and Reaction Engineering (RIPP, SINOPEC) \\ ${ }^{3}$ School of Chemistry and Molecular Engineering, East China University of Science and Technology, \\ Shanghai 200237, China \\ 'yxyang@ecust.edu.cn ${ }^{1}$
}

\begin{abstract}
Ordered 2D hexagonal and parallel arranged pore channel mesoporous silica materials with homogeneous size and spherical shape have been synthesized by using amino acid-based surfactant templating, their ordered mesostructures were characterized by infrared spectroscopy, $\mathrm{X}$-ray diffraction patterns (XRD), scanning electron microscopy (SEM), transmission electron microscopy (TEM) and nitrogen sorption analysis.
\end{abstract}

\section{Introduction}

Recently, synthesis of mesoporous material by the route of self-assemly directed by micelles formed by surfactant has attracted much more interests ${ }^{[1,2]}$. The amino acid-based surfactant is a kind of environmental friendly surfactants based on biological substance, its molecules has amido and carboxyl group in its molecular structure. The positive charge of hydrophilic cations is carried by amido groups, and negative charge is carried by carboxyl groups. This leads to its many excellent functions, such as, excellent detergent action, emulsifiable, anticorrosion and bactericidal ability with low toxicity and high degradability [3]. Until now there is few successful synthesis of ordered mesoporous silica directed by amino acid-based surfactant ${ }^{[4,5]}$.

Due to ordered parallel arranged micelle originating from the amino acid-based surfactant molecules and cosurfactant (3-aminopropyltrimethoxysilan(APS)) by dipole moment and hydrogen bonding, the formation of ordered mesoporous silica is directed by amino acid-based surfactant. The polar amino acid molecules can form hydrogen bonds with NH group of another amino acid molecule; charged groups provoke strong electrostrictive interaction between negative charged carboxyl groups and positive charged amino groups of APS. The presence of a significant dipole moment and hydrogen bonding by amino acids and uneven charge distribution in the molecules causes ordered parallel arranged micelles, which thus directs synthesis of ordered mesoporous silica ${ }^{[6]}$. In this paper, spherical ordered pore

* Corresponding author:yxyang@ecust.edu.cn 
structure was firstly synthesized directed by amino acid-based surfactant, potassium N-dodecyl glycine (MYS-K), and a series of characterizations performed.

\section{Results and Discussion}

\subsection{Reagent}

Potassium N-dodecyl glycine (abbreviated as MYS-K, made in Sigma-Aldrich Co. LLC.), Tetra ethyl orthosilicate(abbreviated as TEOS) and 3-aminopropyltrimethoxysilan (abbreviated as APS, made in Aladdin Industrial Corporation Company Ltd., China).

\subsection{Synthesis of 2 dimensional Hexagonal Mesoporous Silica}

To dissolve $5 \mathrm{~mL}$ potassium N-dodecyl glycine (MYS-K) in $100 \mathrm{~mL}$ deionized water, then add $2 \mathrm{~mL} 3$-aminopropyltrimethoxysilan (APS) and $8.5 \mathrm{~mL}$ Tetra ethyl orthosilicate (TEOS) by magnetic stirring. After $2 \mathrm{~h}$ stirring, the reaction product was divided into two parts, one was transferred to autoclave with polyetrafluoroethylene as lining, followed by hydrothermal treatment at $100^{\circ} \mathrm{C}$ for 2 days, and the solid products were filtrated off, then washed with deionized water and anhydrous ethanol repeatedly, self-dried at room temperature. Finally the product was placed in muffle furnace and calcined at $650^{\circ} \mathrm{C}$ for $6 \mathrm{~h}$, the acquired product was marked as MYS-K-1.

Another part continued to stir for $24 \mathrm{~h}$, the product was filtrated after reaction, and then it was washed with deionized water and anhydrous ethanol repeatedly, self-dried at room temperature. Finally the product was placed in muffle furnace and calcined at $650^{\circ} \mathrm{C}$ for $6 \mathrm{~h}$, the acquired white product was marked as MYS-K-2.

On keeping all the other parameters constant except for MYS-K, the amount of MYS-K was increased to $8 \mathrm{~mL}$, and the experiment was made as above mentioned, the acquired white product was marked as MYS-K-3.

\subsection{Characterization}

The powder X-ray diffraction (XRD) measurements of the mesoporous silica were performed on a Rigaku D/max $2550 \mathrm{VB} / \mathrm{PC}$ X-ray diffractometer using Ni-filtered $\mathrm{Cu} \mathrm{K \alpha}$ radiation $(\lambda=0.15418 \mathrm{~nm})\left[40 \mathrm{~mA}, 40 \mathrm{kV}, 0^{\circ} \sim 6^{\circ}\right.$ of $\left.2 \theta, 1^{\circ}(2 \theta) \cdot \mathrm{min}^{-1}\right]$ at room temperature. The FT-IR spectrogram was recorded by Nicolet Influence 410 infrared spectrometer. The surface morphology and dimensions of the mesoporous silica were observed using a Hitachi s4800 field emission scanning electron microscope with an accelerating voltage of $15.0 \mathrm{kV}$. Pore shape and degree of order of mesoporous silica were observed by a JEOL JEM-2100 transmission electron microscope operating at $200 \mathrm{kV}$ Surface area and pore distribution of mesoporous silica were measured using an ASAP2405N (Micromeritics Instrument Corp., Norcross, GA) physical adsorption apparatus. The surface area was calculated using the Brunauer-Emmett-Teller (BET) method and adsorption data $p / p_{0}$ in the range $0.05 \sim 0.3$. Pore structure distribution of mesoporous silica after calcination is calculated using the Barrett-Joyner-Halenda (BJH) method from adsorption isotherms.

\subsection{Analysis of experimental process}

In the experimental process, white precipitation appeared several minutes after APS and TEOS were added, which shows APS and TEOS hydrolyze much fast. The products marked 
as MYS-K-2 and MYS-K-3 became white powders after calcining, while most of product marked as MYS-K-1 kept cinerous dark after calcining for $6 \mathrm{~h}$, this results show that MYS-K-2 and MYS-K-3 have more connected pore channel with more loose structure, by comparing with MYS-K-1. The MYS-K-1 had not become white powder after calcining, which shows its structure is compact, and leads to pore channel walled up. This is probably because the hydrothermal stability of mesoporous silica synthesized directed by potassium $\mathrm{N}$-dodecyl glycine in this paper is poor, it results in pore structure collapse after hydrothermal treatment.

By experiment, it is also found that, precipitation did not appear after two days if TEOS was selected as sole silicon source. This shows that formation of mesoporous silica, directed by potassium $\mathrm{N}$-dodecyl glycine is related to interaction between anionic headgroup of MYS-K and amino cation connected by $\mathrm{Si}$ atom in APS, the reaction sketch is shown in Fig.1a. In an acidic solution, the $-\mathrm{COO}^{-}$group of $\mathrm{N}$-dodecyl glycine is protonated to a free $-\mathrm{COOH}$ group, and the molecule has an overall positive charge. As the $\mathrm{pH}$ is raised, the $-\mathrm{COOH}$ loses its proton at about $\mathrm{pH} 2$, which represents $\mathrm{pK}_{1}$-the first acid dissociation constant, but above this $\mathrm{pH}$ of 9 , the molecule has an overall negative charge. Amino acid molecules also possess polar groups, which form hydrogen bonds with $\mathrm{NH}$ group of another amino acid molecule; charged groups provoke strong electrostrictive interaction between negative charged carboxyl groups and positive charged amino groups of APS. The presence of a significant dipole moment and hydrogen bonding by amino acids and uneven charge distribution in the molecules causes ordered parallel arranged micelles (Van-der-Waals, dipole-dipole, inductive-orientational interactions)(Shown in Fig.1a), which thus directs synthesis of ordered mesoporous silica.

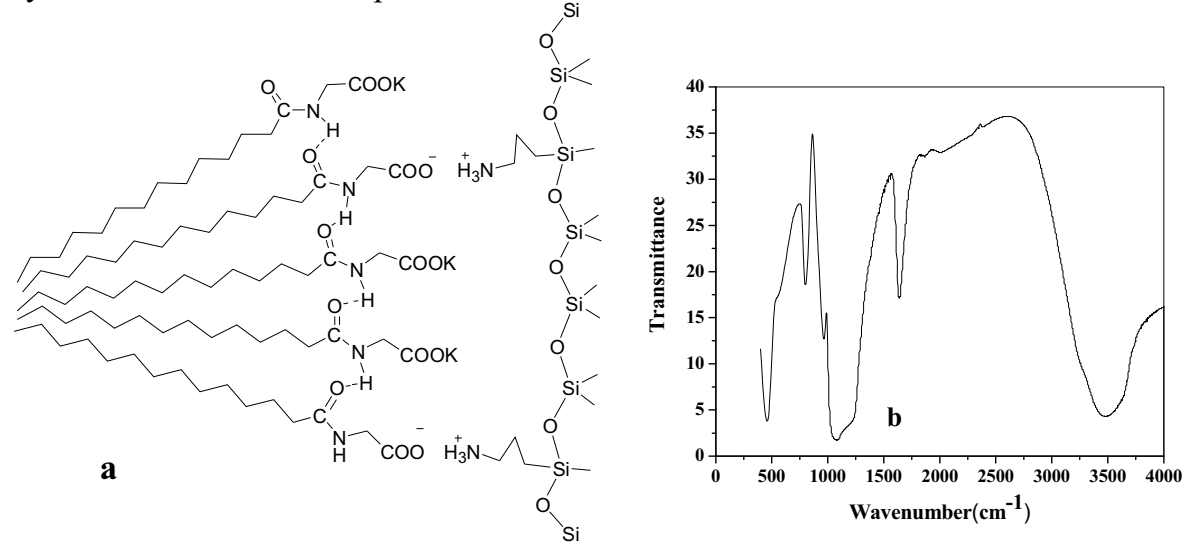

Fig.1 a) Reaction sketch, b) FT-IR patters of MYS-K-2

\subsection{Analysis of infrared spectrum and X-ray diffraction}

The results of infrared spectrum showed, the broad peaks at $3461 \mathrm{~cm}^{-1}$ can be assigned to stretching vibration of $\mathrm{OH}$ group. The strong peaks at $1069 \mathrm{~cm}^{-1}$ can be assigned to asymmetric stretching vibration of $\mathrm{Si}-\mathrm{O}-\mathrm{Si}$, because it is reported that the frequency of the stretching vibration of Si-O-Si bond is $1050 \mathrm{~cm}^{-1}[7]$. The peaks at $963 \mathrm{~cm}^{-1}, 799 \mathrm{~cm}^{-1}, 453$ $\mathrm{cm}^{-1}$ can be assigned to deformation vibration or swing vibration of the Si-O. The peaks at $1643 \mathrm{~cm}^{-1}$ can be assigned to deformation vibration of the H-O-H. The occurrence of the absorption peaks from the vibrations of the $\mathrm{Si}-\mathrm{O}-\mathrm{Si}$, Si-O bond and $\mathrm{O}-\mathrm{H}$ bond in the infrared spectrum indicates that the sample must be $\mathrm{SiO}_{2}$. While there are no any evidence of the peaks related to vibration of the $\mathrm{C}-\mathrm{H}$, which show organic template in mesoporous silica is 
eliminated by high temperature. So the composition of the synthesized materials must be $\mathrm{SiO}_{2}$ according to above mentioned analysis of infrared spectrum.
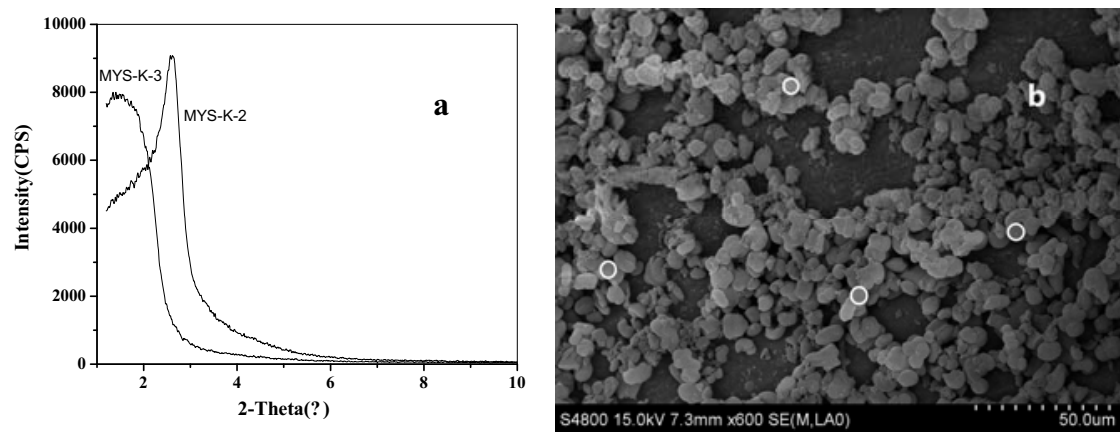

Fig.2 a) SXRD patterns of MYS-K-2 and MYS-K-3, b) SEM images of MYS-K-2

Small angle X-ray diffraction (SXRD) patterns of synthesized MYS-K-2 and MYS-K-3 are shown in Fig.2a. The product MYS-K-2 shows a single well-resolved strong diffraction peak at $2 \theta=2.662^{\circ}$ in the range of $1.2 \sim 10^{\circ}$, which shows a high degree of ordering of the porous structure. This is attributed to typical diffraction peak of mesoporous $\mathrm{SiO}_{2}$, the corresponding d spacing is $3.3 \mathrm{~nm}$. According to Peter T.Tanev ${ }^{[8]}$, higher order Bragg reflections of the hexagonal structure are not resolved, the "single-reflection" products still have short-range hexagonal symmetry, the following TEM images will support this conclusion. So the Bragg reflection of MYS-K-2 can be indexed as [100] assuming P6 symmetry.

The unit cell constant was calculated according to the formula $\alpha_{0}=2 d_{100} / \sqrt{3}$, where $\mathrm{d}_{100}$ is the interplanar spacing corresponding to the [100] Bragg reflection, the acquired $\mathrm{a}_{0}$ calculation value is $3.8 \mathrm{~nm}$. The product MYS-K-3 shows a weak and broad diffraction peak at $2 \theta=1.46^{\circ}$ in the range of $1.2 \sim 10^{\circ}$, which shows a lower degree of ordering of the porous structure by comparing with MYS-K-2 product, as seen from the following TEM images.

\subsection{Scanning electron microscopy and transmission electron microscopy}

The SEM image of MYS-K-2 is clearly shown in Fig.2b, MYS-K-2 product consists of well dispersed, homogeneous and spherical shaped particles of about $5 \mu \mathrm{m}$ in diameter.

Fig.3a and Fig.3b represent TEM images of MYS-K-2 along two different directions respectively. TEM image recorded in Fig.3a can be indexed as [100] direction according to the results of XRD, the image clearly shows ordered 2D hexagon mesostructured with homogeneous pores arranged, the pore size was estimated as about $2.1 \mathrm{~nm}$. Fig. $3 \mathrm{~b}$ is the TEM image recorded in [110] direction, the image clearly shows ordered parallel arranged pore channel structure, the distance between two pore center was estimated as $3.6 \mathrm{~nm}$ from TEM images, it is in good agreement with the value calculated from XRD data $\left(\mathrm{a}_{0}\right.$ calculation value is $3.8 \mathrm{~nm}$ ), so the pore wall thickness can be estimated as $1.5 \mathrm{~nm}$ from the distance and the pore size. 

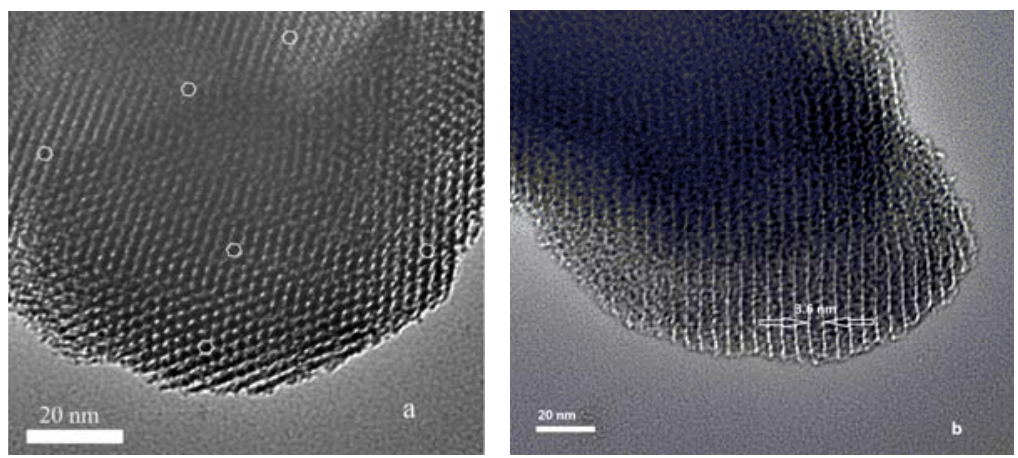

Fig.3 TEM images of MYS-K-2 along (a)[100],(b)[110] directions

\subsection{Nitrogen sorption measurement}

$\mathrm{N}_{2}$ sorption isotherm of MYS-K-2 is of type IV at relative pressure $p / p_{0}$ of 0.01 0.99(Fig.4a), indicating the presence of mesopores. An unobvious upward at relative pressure $\mathrm{p} / \mathrm{p}_{0}$ of $0.01 \sim 0.05$ is also observed, demonstrating the presence of a small quantity of mesoporosity. A linear sorption isotherm is observed at relative pressure $\mathrm{p} / \mathrm{p}_{0}$ of $0.05 \sim 0.35$, according to the saturated adsorption amount is obtained by slope and intercept of line, so specific surface area of MYS-K-2 is acquired as $1108 \mathrm{~m}^{2} / \mathrm{g}$. The adsorption isotherm is characterized by an obvious increase in the amount adsorbed in the range of relative pressure $\mathrm{p} / \mathrm{p}_{0}$ of $0.30 \sim 0.35$, which is typical of the filling volume of mesopores. When the relative pressure $\mathrm{p} / \mathrm{p}_{0}$ exceeds 0.35 , the sorption gets equilibrium, sorption isotherm of MYS-K-2 get smooth. The pore size distribution of MYS-K-2 is seen from Fig.4b, a narrow pore size is observed, the mesopore size is calculated to be $2.2 \mathrm{~nm}$, which is in a good agreement with the value estimated by TEM image.
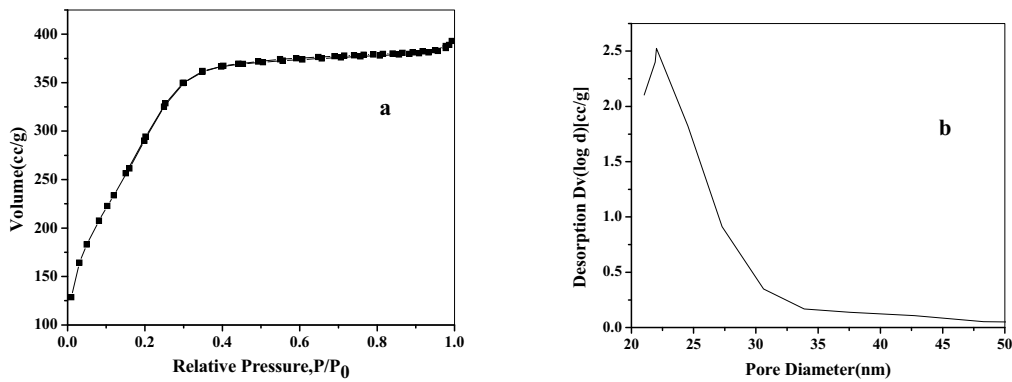

Fig.4 a) Sorption isotherm of MYS-K-2, b)The pore size distribution of MYS-K-2

\section{Summary}

In this paper, homogeneous size and spherical shaped mesoporous silica of about $5 \mu \mathrm{m}$ in diameter was firstly synthesized according to a route directed by an environment-friendly, mild and biodegradable amino acid-based surfactant, potassium N-dodecyl glycine with 3-aminopyltrimethoxysilan and TEOS as silicon source in absence of any inorganic acids. TEM images clearly show ordered parallel arranged pore channel structure along [110], and ordered hexagon mesostructured with homogeneous pores arranged along [100] direction, the distance between different pore center is $3.6 \mathrm{~nm}$, in good agreement with the value 
estimated from XRD. Type of sorption isotherm belongs to typical IV adsorption, specific surface area is $1108 \mathrm{~m}^{2} / \mathrm{g}$ measured by BET. The pore size distribution is narrow with a pore size of about $2.2 \mathrm{~nm}$.

\section{Acknowledgement}

The authors are grateful to the financial support from state key laboratory of catalytic materials and reaction engineering (RIPP, SINOPEC).

\section{References}

1. Wang D C, Mesoporous aluminium oxide support with large pore volume by nano self-assembly, Science China.39 (2009) 420-431.

2. Mohammad N F, Othman R, Yeoh F Y, Pore Characteristics of Mesoporous Carbonated Hydroxyapatite Synthesised with Different Nonionic Surfactant and Carbonate Concentration, Materials Science Forum.819 (2015) 353-360.

3. Che S, Garcia-bennett A E, Yokoi, et al. A novel anionic surfactant templating route for synthesizing mesoporous silica with unique structure, Nature materials. 2 (2003) 801-805.

4. Yang Y X, Huang Z, Deng W J, etal, Synthesis of mesoporous silica templated by Sodium N-dodecyl glycine, Microporous and Mesoporous Materials. 116 (2008) 267-276.

5. B Thomas, $\mathrm{N}$ Baccile, $\mathrm{S}$ Masse, $\mathrm{C}$ Rondel, et al. Mesostructured silica from amino acid-based surfactant formulations and sodium silicate at neutral $\mathrm{pH}$, Journal of Sol-Gel Science and Technology. 58 (2011) 170-174.

6. Liu H T, Yang Y X, Liu L, Yuan H M, Chen C, Liu X N, Effects of different chain lengths and chiral branched amino acids on the synthesis of mesoporous silica, Journal of nanoscience and nanotechnology. 14 (2014) 5518-5528.

7. Wang C,Y, et al. Synthesis and characterization of organophilic montmorillonite, Natural science Journal of harbin normal university. 19 (2003) 63-65.

8. Tanev P T, Pinnavaia T J. A neutral templating route to mesoporous molecular sieves, Science. 267 (1995) 865-867. 